\title{
GENERALIZED AERODYNAMIC FORCES ON A FLEXIBLE CYLINDRICAL SHELL UNDERGOING TRANSIENT MOTION*
}

BY

E. H. DOWELL

Princeton University

Nomenclature

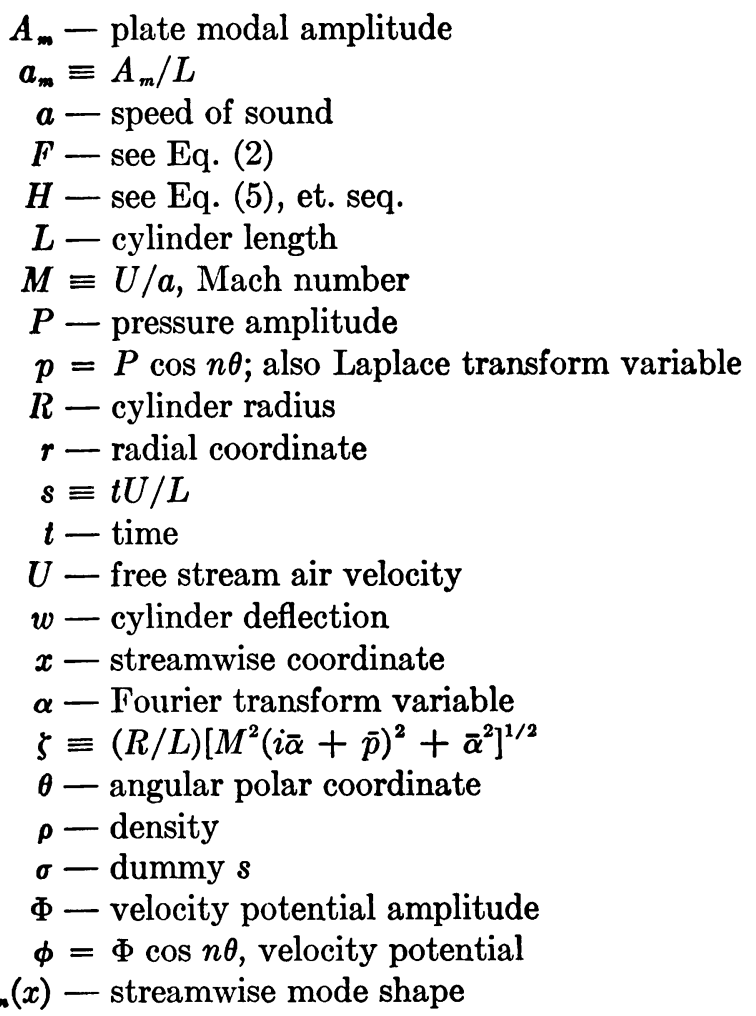

Superscripts

* - Laplace transform with respect to time

$\dagger$ - Fourier transform with respect to streamwise coordinate

- - derivative with respect to time-like argument

' - derivative with respect to space-like argument

Subscripts

$R$ - real part

$I$ - imaginary part

*Received August 23, 1967; revised manuscript received November 2, 1967. 
1. Introduction. There are two rather closely related problems involving the motion of a flexible cylindrical shell in a fluid flow which are of current engineering interest. These are the stability of the fluid shell system (shell or panel flutter) and the response of the system to "external" forces, e.g., response to pressure fluctuations in a turbulent boundary layer. In either case, the determination of the aerodynamic forces due to the shell motion is of interest. Heretofore only the special case of sinusoidal or simple harmonic motion has been treated in a series of papers by Widnall and the author [1-3] for both external and internal flow. This work was, in turn, based on earlier studies by Randall [4] and Stearman [5]. In the present work the more general case of arbitrary time (as well as spatial) dependence of the shell motion is considered. The approach used is analogous to that previously employed for the flat plate [6]. Only the external flow case is treated.

2. Problem formulation and solution. A flexible cylindrical shell of finite length undergoing arbitrary temporal and spatial motion is considered. There is an external, inviscid, irrotational flow parallel to the axis of the cylinder; the object is to determine the fluid (aerodynamic) forces acting on the shell. In order to make the problem mathematically tractable for subsonic flow, $M<1$, the finite length flexible shell is considered to be a portion of an infinitely long cylinder, the remainder of which is rigid. For supersonic flow, $M>1$, the solution is not as restrictive since the flow behind the flexible shell cannot affect the flow over it, and that in front of the shell need only be uniform and parallel as it arrives at the flexible shell.

Within the framework of linear theory, the boundary value-initial value problem may be stated as follows: We seek a solution to the partial differential equation for the velocity potential $\phi$

$$
\nabla^{2} \phi-\left(1 / a^{2}\right)[\partial / \partial t+U \partial / \partial x]^{2} \phi=0
$$

subject to the boundary condition

$$
\begin{aligned}
\left.\frac{\partial \phi}{\partial r}\right|_{r=R} & =U \frac{\partial w}{\partial x}+\frac{\partial w}{\partial t} \equiv F(x, t) \cos n \theta \text { on flexible shell } \\
& =0 \text { on rigid cylinder }
\end{aligned}
$$

and also an appropriate boundary condition as $r \rightarrow \infty$. The Fourier decomposition in the circumferential variable $\theta$ may be appropriately summed to treat an arbitrary variation of shell deflection $w$ with $\theta$.

This problem will be treated by the transform calculus, employing a Laplace transform with respect to time and a Fourier transform with respect to the spatial variable $x$. Let

$$
\phi(x, r, \theta, t)=\Phi(x, r, t) \cos n \theta
$$

and define

$$
\Phi^{*}(x, r ; p) \equiv \int_{0}^{\infty} e^{-p t} \Phi(x, r, t) d t
$$

and

$$
\Phi^{*^{\dagger}}(r ; \alpha, p) \equiv \int_{-\infty}^{\infty} e^{-i \alpha x} \Phi^{*}(x, r ; p) d x
$$


Equations (1) and (2) become (assuming that $\phi=\partial \phi / \partial t=0$ at $t=0^{-}$)

$$
\frac{d^{2} \Phi^{*^{\dagger}}}{d r^{2}}+1 / r \frac{d \Phi^{*^{\dagger}}}{d r}-\frac{n^{2}}{r^{2}} \Phi^{*^{\dagger}}-\mu^{2} \Phi^{*^{\dagger}}=0
$$

where

$$
\mu \equiv\left[\underline{p}^{2}+2 \frac{M p i \alpha}{a}-\alpha^{2}\left(M^{2}-1\right)\right]^{1 / 2}
$$

and

$$
\left.\frac{d \Phi^{*}}{d r}\right|_{r=R}=F^{*}
$$

with

$$
F^{*^{\dagger}} \equiv \int_{0}^{\infty} \int_{-\infty}^{\infty} e^{-p t} e^{-i \alpha x} F d x d t
$$

Solving Eq. (3) subject to (4) gives

$$
\left.\Phi^{*^{\dagger}}\right|_{r=R}=R \frac{K_{n}(\zeta)}{\zeta K_{n}^{\prime}(\zeta)} F^{*^{\dagger}}
$$

where

$$
\begin{aligned}
& \zeta \equiv(R / L)\left[M^{2}(i \bar{\alpha}+\bar{p})^{2}+\bar{\alpha}^{2}\right]^{1 / 2}, \\
& \bar{\alpha} \equiv \alpha L, \quad \bar{p} \equiv p L / U .
\end{aligned}
$$

(The square root of $\zeta$ is to be chosen such that the condition of finiteness or radiation is satisfied at infinity. We will not need to do this explicitly.) Using the convolution and inversion theorems we have

$$
\Phi_{r=R}^{\dagger}=R \int_{0} H(s-\sigma) F^{\dagger}(\sigma) d \sigma
$$

where

$$
F^{\dagger}(\sigma) \equiv \int_{-\infty}^{\infty} F(\sigma, \bar{x}) e^{-i \bar{\alpha} \bar{x}} d \bar{x}
$$

and

$$
\begin{gathered}
H(s-\sigma) \equiv \frac{1}{2 \pi i} \int_{-i \infty}^{i \infty} \frac{K_{n}(\zeta)}{\zeta K_{n}^{\prime}(\zeta)} e^{\bar{p}(x-\sigma)} d \bar{p} \\
\bar{x} \equiv x / L, \quad s \equiv t U / L .
\end{gathered}
$$

Formally the inversion may now be made to the spatial domain. However, instead we proceed with the calculation of the Fourier transform of the fluid forces. The fluid pressure is related to the velocity potential by the Bernoulli formula,

$$
p=-\rho[\partial \phi / \partial t+U \partial \phi / \partial x] \text {. }
$$

From Eqs. (5) and (6) one may compute the Fourier transform of the pressure amplitude as 


$$
P_{r=R}^{\dagger}=-\rho U(R / L)\left\{H(0) F^{\dagger}(s)+\int_{0}^{s}[i \bar{\alpha} H(s-\sigma)+\dot{H}(s-\sigma)] F^{\dagger}(\sigma) d \sigma .\right.
$$

By noting that

$$
H(0)=\lim _{\bar{p} \rightarrow \infty} \bar{p} \frac{K_{n}(\zeta)}{\zeta K_{n}^{\prime}(\zeta)}=\frac{-1}{(R / L) M}
$$

we see the well known result that at $s=0^{+}$the pressure is that given by "piston theory" [7]. Again a formal inversion into the spatial domain may be made if desired.

For the usual applications, the forces of interest are the "generalized aerodynamic forces" rather than the pressure itself. If

$$
\bar{w}(x, t)=\sum_{m} A_{m}(t) \psi_{m}(x),
$$

where $w(x, \theta, t) \equiv \bar{w}(x, t) \cos n \theta$, then the (nondimensional) generalized force, $Q_{m r}$, is defined as

$$
Q_{m r}=\frac{\int_{0}^{L} P_{m} \psi_{r}(x) d x}{\rho U^{2} L}
$$

where $P_{m}$ is the pressure amplitude due to

$$
\bar{w}=A_{m}(t) \psi_{m}(x) .
$$

A considerable economy of effort may be achieved by performing the integral over $x$ in Eq. (9) before inverting the Fourier transform. Having done this, $Q_{m}$ may be written

$$
Q_{m r}=a_{m}(s) S_{m r}+\dot{a}_{m} D_{m r}+\int_{0}^{\bullet} a_{m}(\sigma) H_{m r}(s-\sigma) d \sigma+\int_{0}^{\bullet} \dot{a}_{m}(\sigma) I_{m r}(s-\sigma) d \sigma
$$

where

$$
\begin{aligned}
S_{m r} & \equiv \frac{1}{M} \int_{0}^{1} \psi_{m}^{\prime}(\xi) \psi_{r}(\xi) d \xi \\
D_{m r} & \equiv \frac{1}{M} \int_{0}^{1} \psi_{m}(\xi) \psi_{r}(\xi) d \xi \\
H_{m r} & \equiv-\left(\frac{R}{L}\right) \frac{1}{2 \pi} \int_{-\infty}^{\infty} i \bar{\alpha}[i \bar{\alpha} H(s-\sigma, \bar{\alpha})+\dot{H}(s-\sigma, \bar{\alpha})] G_{m r}(\bar{\alpha}) d \bar{\alpha} \\
I_{m r} & \equiv-\left(\frac{R}{L}\right) \frac{1}{2 \pi} \int_{-\infty}^{\infty}[i \bar{\alpha} H(s-\sigma, \bar{\alpha})+\dot{H}(s-\sigma, \bar{\alpha})] G_{m r}(\bar{\alpha}) d \bar{\alpha}, \\
G_{m r} & \equiv \int_{0}^{1} \psi_{m}(\xi) e^{-i \bar{\alpha} \xi} d \xi \int_{0}^{1} \psi_{r}(\xi) e^{i \bar{\alpha} \xi} d \xi .
\end{aligned}
$$

In the derivation it has been assumed that $\psi_{m}(0)=\psi_{m}(1)=0$. For the commonly used $\psi_{m}, S_{m r}, D_{m r}$, and $G_{m r}$ may be integrated analytically; $H_{m r}$ and $I_{m r}$ must be determined by numerical integration. In order to do this, it will be necessary to develop $H$ in a more explicit form. 
Development of $H$. Recall

Define

$$
H(s-\sigma) \equiv \frac{1}{2 \pi i} \int_{-i \infty}^{i \infty} \frac{K_{n}(\zeta)}{\zeta K_{n}^{\prime}(\zeta)} e^{\bar{j}(\varepsilon-\sigma)} d \bar{p}
$$

then

$$
\epsilon \equiv(R / L) M[\bar{p}+i \bar{\alpha}]
$$

$$
\zeta^{2}=\epsilon^{2}+[(R / L) \bar{\alpha}]^{2}
$$

and, using a well known inversion formula [8],

$$
H(s-\sigma)=\frac{L}{R M} e^{-i \bar{\alpha}(\bullet-\sigma)}\left\{\frac{1}{2 \pi i} \int_{-i \infty}^{i \infty} e^{(\epsilon L / R M)(\bullet-\sigma)} \frac{K_{n}(\zeta)}{\zeta K_{n}^{\prime}(\zeta)} d \epsilon\right\} .
$$

Now Randall has evaluated the following functions

$$
V(s)=-\frac{1}{2 \pi i} \int_{-i \infty}^{i \infty} e^{\epsilon \epsilon} \frac{K_{n}(\epsilon)}{\epsilon K_{n}^{\prime}(\epsilon)} d \epsilon .
$$

Using another known inversion formula, ([8, p. 227, No. 5]) $H$ may be written in terms of the Randall functions, $V$.

$$
H(s)=\frac{-L}{R M} e^{-i \bar{\alpha} s}\left\{V\left(\frac{L}{R} \frac{S}{M}\right)-\frac{\bar{\alpha}}{M} \int_{0}^{s} J_{1}\left(\frac{\bar{\alpha} u}{M}\right) V\left[\frac{L}{R M}\left(s^{2}-u^{2}\right)^{1 / 2}\right] d u\right\} .
$$

The above evaluation of $H$ follows very closely a similar calculation of Stearman [5] who treated the problem of a harmonically oscillating cylinder by using a Laplace transform with respect to $x$. It turns out that his procedure is much more conveniently employed with respect to the time variable, as indicated here.

The Randall functions have several desirable properties among which are

1. They are real; $H$, on the other hand, is complex by virtue of the complex exponential $e^{-i \bar{\alpha} e}$.

2. $V$ only depends on a single argument and one parameter, $n$. See Eq. (12).

3. $V$ has been tabulated for $n=0-10$. For the present work, its derivative has also been tabulated using the computation procedure suggested by Randall [4].

Other interesting properties of $V$ are

4. $V(0)=\lim _{\theta \rightarrow \infty}\left\{-\frac{K_{n}(\epsilon)}{K_{n}^{\prime}(\epsilon)}\right\}=1$

Similarly $V(0)=-.5$ for all $n$

5. $V(\infty)=\lim _{\epsilon \rightarrow 0}\left\{\frac{-K_{n}(\epsilon)}{K_{n}^{\prime}(\epsilon)}\right\}=0$

Similarly $\dot{V}(\infty)=0$

6. $\int_{0}^{\infty} \dot{V}(s) d s=-1$ from 4 and 5

7. $\int_{0}^{\infty} V(s) d s=\frac{1}{2 \pi i} \int_{-i \infty}^{i \infty} \frac{1}{\epsilon}\left\{\frac{-K_{n}(\epsilon)}{\epsilon K_{n}^{\prime}(\epsilon)}\right\}\left[\left.e^{p \infty}\right|_{0-\infty}-\left.e^{p \infty}\right|_{0-0}\right] d \epsilon$

$$
=\lim _{\epsilon \rightarrow 0}\left\{\frac{-K_{n}(\epsilon)}{\epsilon K_{n}^{\prime}(\epsilon)}\right\}-\lim _{\epsilon \rightarrow \infty}\left\{\frac{-K_{n}(\epsilon)}{\epsilon K_{n}^{\prime}(\epsilon)}\right\}=1 / n .
$$


This last property combined with the fact that $V$ decays with its argument more rapidly as $n$ increases gives $V$ a pseudo delta function behavior for large $n$ (or large $L / R$ ).

Now consider $\dot{H}$ and, more specifically, $i \bar{\alpha} H+\dot{H}$ which we need for $H_{m}$, and $I_{m r}$. From Eq. (13)

$$
\begin{aligned}
i \bar{\alpha} H(s)+\dot{H}(s)= & \frac{L}{R} \bar{M}^{2} e^{-i \bar{\alpha} s}\left\{-\frac{L}{R} \dot{V}\left(\frac{L}{R M} s\right)+\bar{\alpha} J_{1}\left(\frac{\bar{\alpha} s}{M}\right)\right. \\
& \left.+\frac{L}{R} \frac{\bar{\alpha}}{M} \int_{0}^{s} J_{1}\left(\frac{\bar{\alpha} u}{M}\right) \dot{V}\left[\frac{L}{R M}\left(s^{2}-u^{2}\right)^{1 / 2}\right] \frac{s}{\left(s^{2}-u^{2}\right)^{1 / 2}} d u\right\} .
\end{aligned}
$$

It will be noted that the last term contains an integrable square root singularity as $u \rightarrow s$. For numerical work, it is desirable to remove this by an obvious change of variable, viz.,

$$
\begin{aligned}
K(s) \equiv i \bar{\alpha} H(s)+\dot{H}(s) & =\frac{L}{R M} e^{-i \bar{\alpha} s}\left\{-(L / R) \dot{V}\left(\frac{L}{R} \bar{M} s\right)+\bar{\alpha} J_{1}\left(\frac{\bar{\alpha} s}{M}\right)\right. \\
+ & \left.\frac{L}{R} \frac{\bar{\alpha}}{M} \int_{0}^{s} J_{1}\left[\frac{\bar{\alpha}}{M}\left(s^{2}-u^{2}\right)^{1 / 2}\right] \dot{V}\left[\frac{L}{R} \frac{L}{M} u\right] \frac{s}{\left(s^{2}-u^{2}\right)^{1 / 2}} d u\right\} .
\end{aligned}
$$

Note that $\lim _{n \rightarrow 0} J_{1}(s) / s$ is finite.

Finally, using Eq. (15) and noting the even and odd properties of $G_{m r}$ and $K$ with respect to $\bar{\alpha}, H_{m r}$ and $I_{m r}$ may be written

$$
\begin{aligned}
H_{m r}(s) & =-\frac{1}{\pi} \frac{1}{M^{2}} \int_{0}^{\infty} \bar{\alpha}\left[G_{m r_{R}} \sin \bar{\alpha} s-G_{m r} \cos \bar{\alpha} s\right] T(\bar{\alpha}) d \bar{\alpha} \\
I_{m r}(s) & =-\frac{1}{\pi} \frac{1}{M^{2}} \int_{0}^{\infty}\left[G_{m r_{R}} \cos \bar{\alpha} s+G_{m r_{r}} \sin \bar{\alpha} s\right] T(\bar{\alpha}) d \bar{\alpha}
\end{aligned}
$$

where

$$
\begin{aligned}
T(\bar{\alpha}) \equiv \bar{\alpha} J_{1}\left(\frac{\bar{\alpha} s}{M}\right)-(L / R) \dot{V}\left(\frac{L}{R} \frac{s}{M}\right) & \\
& +(L / R) \frac{\bar{\alpha}}{M} \int_{0}^{s} J_{1}\left[\frac{\bar{\alpha}}{M}\left(s^{2}-u^{2}\right)^{1 / 2}\right] \dot{V}\left[\frac{L}{R} \frac{u}{M}\right] \frac{s}{\left(s^{2}-u^{2}\right)^{1 / 2}} d u .
\end{aligned}
$$

For $L / R \rightarrow 0, T(\bar{\alpha}) \rightarrow \bar{\alpha} J_{1}(\bar{\alpha} s / M)$ and we recover the result for a two-dimensional flat plate [6]. Hence the above form will be termed the two-dimensional approach.

$H_{m r}$ and $I_{m r}$ are "admittance functions" which may be evaluated once and for all.

Slender Body Approach. There is an alternate formulation which is of some physical and mathematical significance which we briefly consider here. By interchanging the convolution argument, Eq. (7) may be written

$$
P^{\dagger}=-\rho U \frac{R}{L}\left\{F^{\dagger}(0) H(s)+\int_{0}^{s}\left[\dot{F}^{\dagger}(s-\sigma)+i \bar{\alpha} F^{\dagger}(s-\sigma)\right] H(\sigma) d \sigma\right\} .
$$

Proceeding as before and omitting details, $Q_{m r}$ may be written as

$$
\begin{aligned}
Q_{m r}=\frac{1}{M}\left\{a_{m}(0) X_{m r}(s)+\dot{a}_{m}(0) Y_{m r}(s)\right. & +\int_{0}^{s} \ddot{a}_{m}(\sigma) Y_{m r}(s-\sigma) d \sigma \\
& \left.+2 \int_{0}^{s} \dot{a}_{m}(\sigma) X_{m r}(s-\sigma) d \sigma+\int_{0}^{s} a_{m}(\sigma) Z_{m r}(s-\sigma) d \sigma\right\}
\end{aligned}
$$


where

$$
\begin{aligned}
X_{m r}(s) & \equiv \frac{1}{\pi} \int_{0}^{\infty} \bar{\alpha}\left[G_{m r_{R}} \sin \bar{\alpha} s-G_{m r_{I}} \cos \bar{\alpha} s\right] T_{1}(\bar{\alpha}) d \bar{\alpha} \\
Y_{m r}(s) & \equiv \frac{1}{\pi} \int_{0}^{\infty}\left[G_{m r_{k}} \cos \bar{\alpha} s+G_{m r_{I}} \sin \bar{\alpha} s\right] T_{1}(\bar{\alpha}) d \bar{\alpha} \\
Z_{m r}(s) & \equiv-\frac{1}{\pi} \int_{0}^{\infty} \bar{\alpha}^{2}\left[G_{m r_{R}} \cos \bar{\alpha} s+G_{m r_{I}} \sin \bar{\alpha} s\right] T_{1}(\bar{\alpha}) d \bar{\alpha}
\end{aligned}
$$

and

$$
T_{1}(\bar{\alpha}) \equiv V\left(\frac{L}{R} \frac{s}{M}\right)-\frac{\bar{\alpha}}{M} \int_{0}^{s} J_{1}\left(\frac{\bar{\alpha} u}{M}\right) V\left[\frac{L}{R M}\left(s^{2}-u^{2}\right)^{1 / 2}\right] d u .
$$

One may show that

$$
\begin{aligned}
& \frac{X_{m r}}{M}(0)=S_{m r}=\frac{1}{M} \int_{0}^{1} \psi_{m}^{\prime}(\xi) \psi_{r}(\xi) d \xi, \\
& \frac{Y_{m r}}{M}(0)=D_{m r}=\frac{1}{M} \int_{0}^{1} \psi_{m}(\xi) \psi_{r}(\xi) d \xi,
\end{aligned}
$$

hence at $s=0$ the results are still those of "piston theory". Also as $n$ becomes large or $L / R$ increases (for $n \neq 0$ ) the $X_{m r}, Y_{m r}, Z_{m r}$ are pseudo delta functions so that

$Q_{m r} \rightarrow \frac{R}{L} \frac{1}{n}\left\{\ddot{a}_{m}(s) \int_{0}^{1} \psi_{m}(\xi) \psi_{r}(\xi) d \xi+2 \dot{a}_{m}(s) \int_{0}^{1} \psi_{m}^{\prime}(\xi) \psi_{r}(\xi) d \xi+a_{m}(s) \int_{0}^{1} \psi_{m}^{\prime \prime}(\xi) \psi_{r}(\xi) d \xi\right\}$.

This is the "slender body" limit which has been previously discussed for simple harmonic motion [1]-[3] generalized to arbitrary time-dependent motion.

3. Discussion. Perhaps a brief word is in order with regard to the motivation for the manner in which the several integrations were carried out. The object, of course, is to minimize the amount of numerical work required. With the present solution, using either the two-dimensional or slender body approach, a double numerical integration is required. First either $T$ or $T_{1}$ is evaluated and then $H_{m r}$ and $I_{m r}$ or $X_{m r}, Y_{m r}$, and $Z_{m r}$ are calculated. Between the two options, two-dimensional vs. slender body approach, there is little to choose, in general; though, as one would expect, for small $L / R$ the twodimensional approach is somewhat more convenient, while for $L / R$ large the converse is true.

There are at least two other distinct approaches to the problem which are worthy of mention.

(i) A solution is available for simple harmonic motion [1]-[3]. In principle, this solution could be used to obtain the arbitrary time-dependent solution by a simple Fourier superposition over all frequencies. Preliminary investigation of this procedure suggested it is less accurate and/or efficient than the method used here.

(ii) An integral form can be derived expressing $\Phi$ or $P$ in terms of the distribution of $F$ over the shell from which $\Phi$ or $P$ could be evaluated by a numerical quadrature [7]. This approach is commonly used for oscillating lifting surfaces. However, it is less efficient than the transform approach when $F$ is known everywhere, i.e. one has an ordinary rather than a mixed boundary value problem. 
4. Numerical results. Representative results will be presented for a family of shell deformations,

$$
\psi_{m}(\xi)=\sin m \pi \xi \quad m=1,2, \cdots
$$

The time dependence will be taken as a unit step function,

$$
\begin{aligned}
a_{m}(s) & =1 \text { for } s>0 \\
& =0 \text { for } s<0
\end{aligned}
$$

In Figs. 1 and 2 are presented results for $Q_{11}$ and $Q_{12}$ for $M=1.414, L / R=2$ and various $n$. In Fig. 3 and 4, results are shown for $L / R=2, n=0$, various $M$ and in Fig. 5 and 6 for $L / R=2, n=10$, various $M$. These data have a number of interesting features among which are:

(i) The $s=0^{+}$results are those given by the piston theory [7] and are independent of $L / R$ and $n$.

(ii) For supersonic flow, the fluid has a finite memory of duration $M /(M-1)$ and thus for $s>M /(M-1)$ the values of $Q_{m r}$ do not change.

(iii) As $n$ (and $L / R$ ) increase the generalized forces reach their asymptotic (large $s$ ) values more quickly. For large $n$ (and large $L / R$ for $n \neq 0$ ) the aerodynamic forces are of the "slender body" type.

(iv) Conversely, for small $n$ (and $L / R$ ) the aerodynamic forces are nearly twodimensional.

(v) For $n=0, M=1.0$, the aerodynamic forces become indefinitely large as $s \rightarrow \infty$. For $n \neq 0$, the aerodynamic forces remain finite and, for $L / R \neq 0$, approach the slender body values.

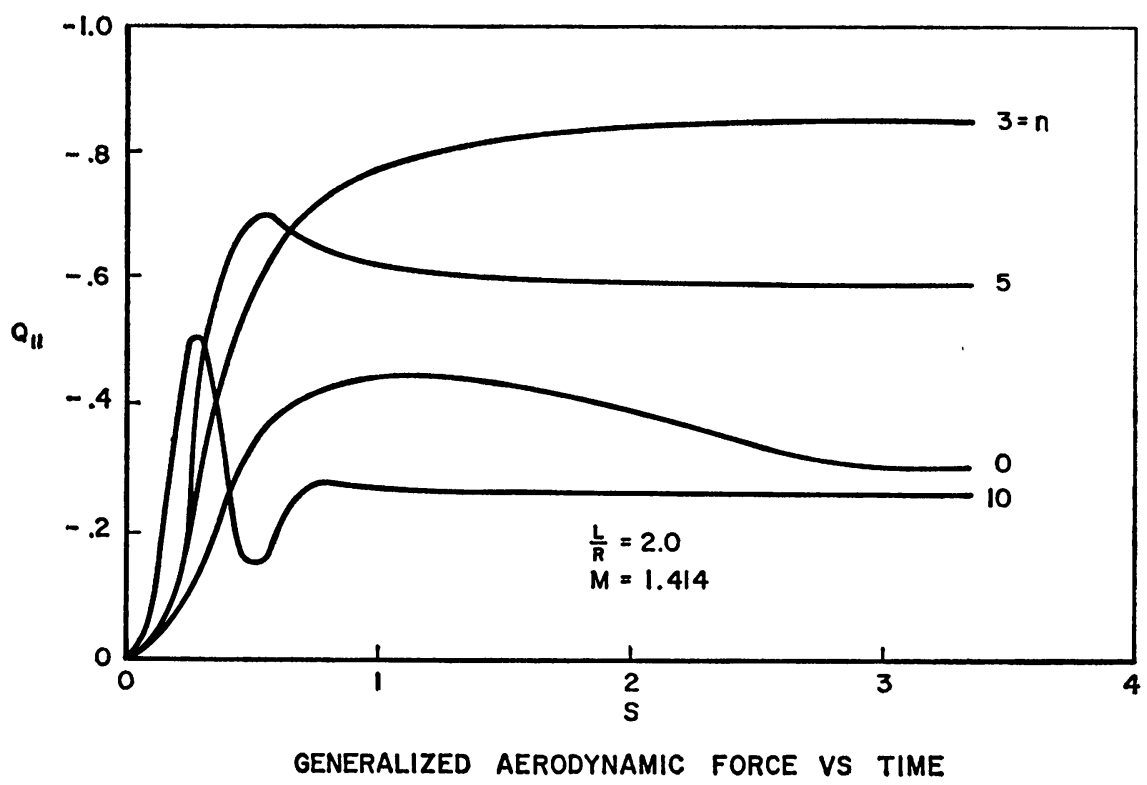



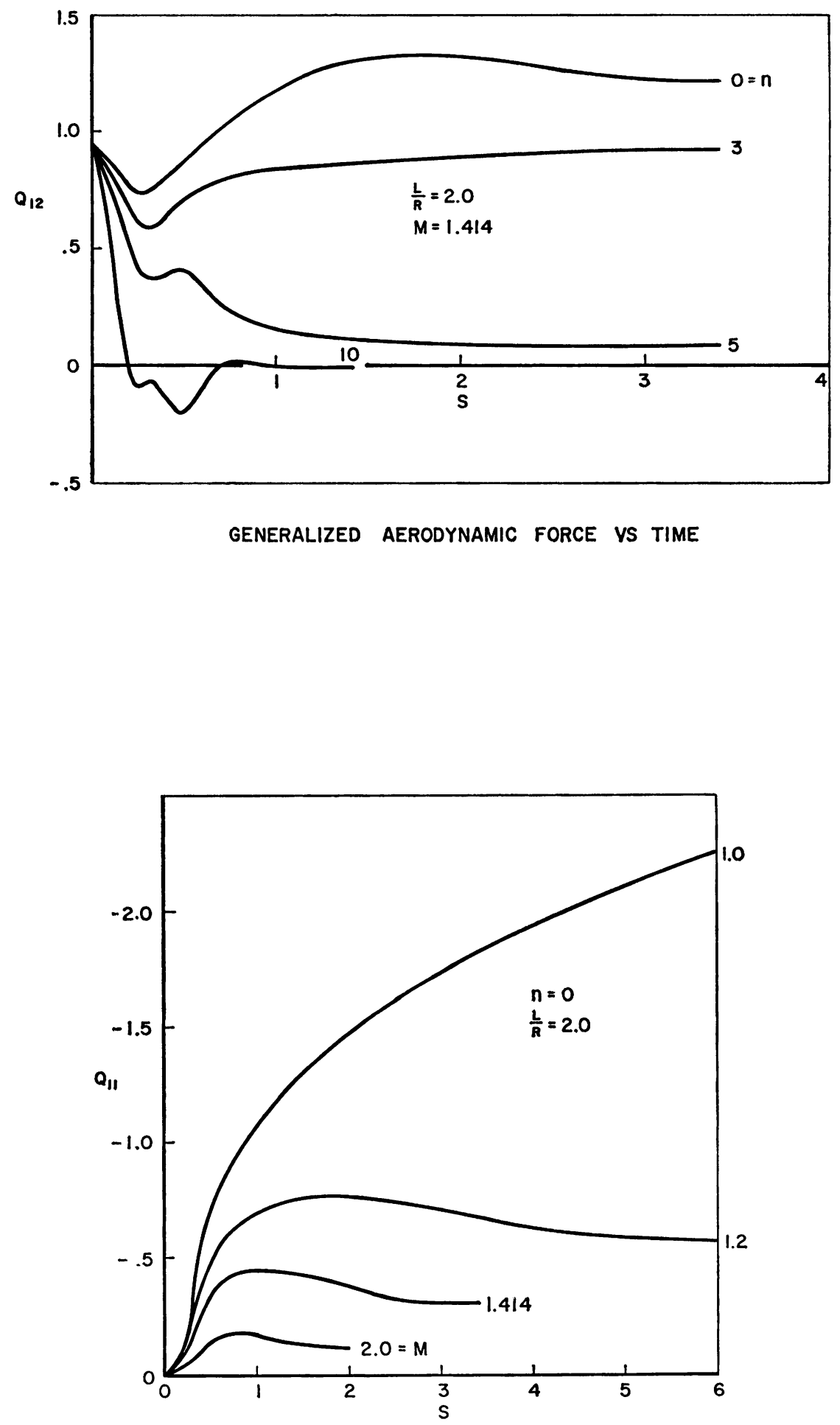

GENERALIZED AERODYNAMIC FORCE VS TIME 


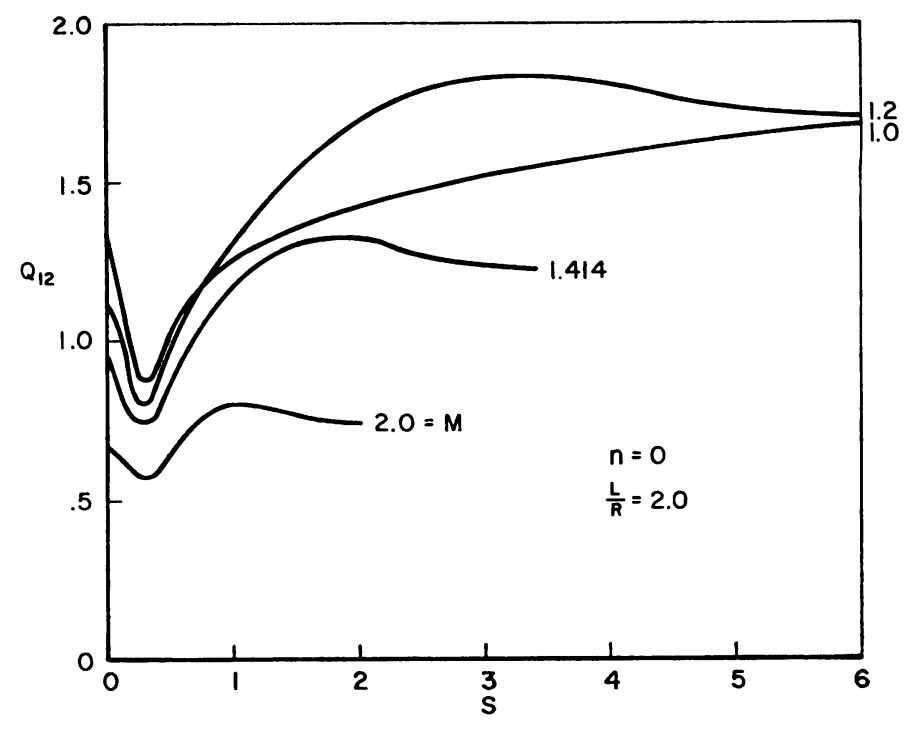

GENERALIZED AERODYNAMIC FORCE VS TIME

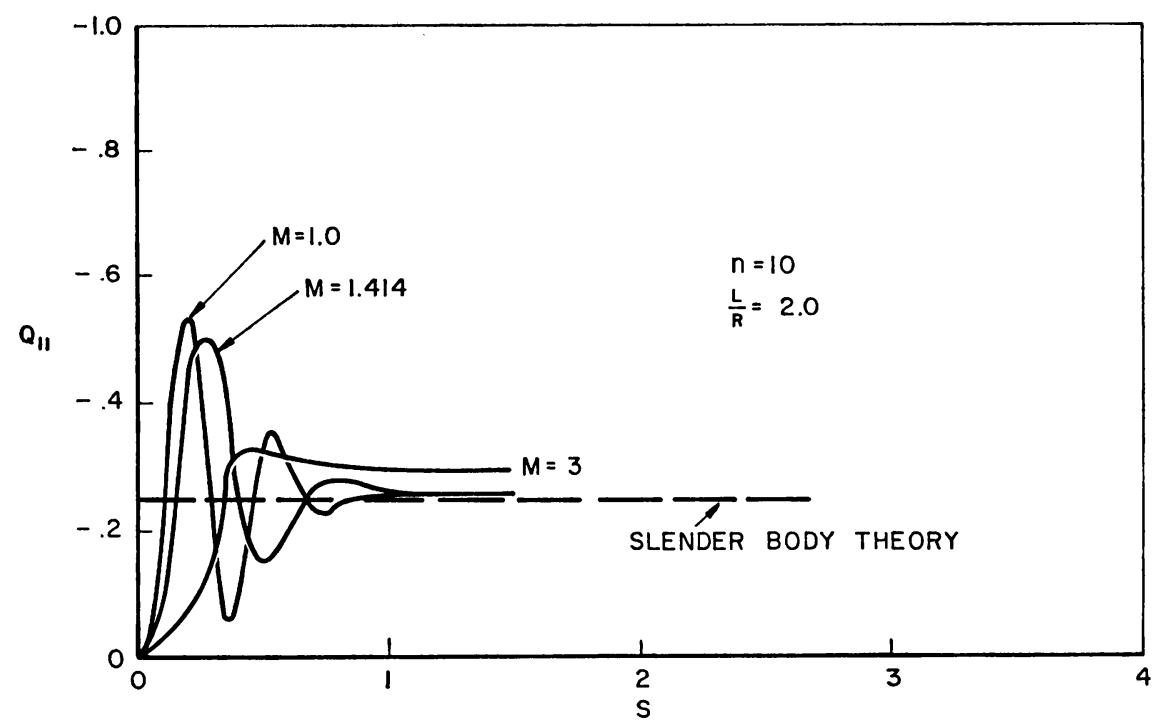

GENERALIZED AERODYNAMIC FORCE VS TIME 


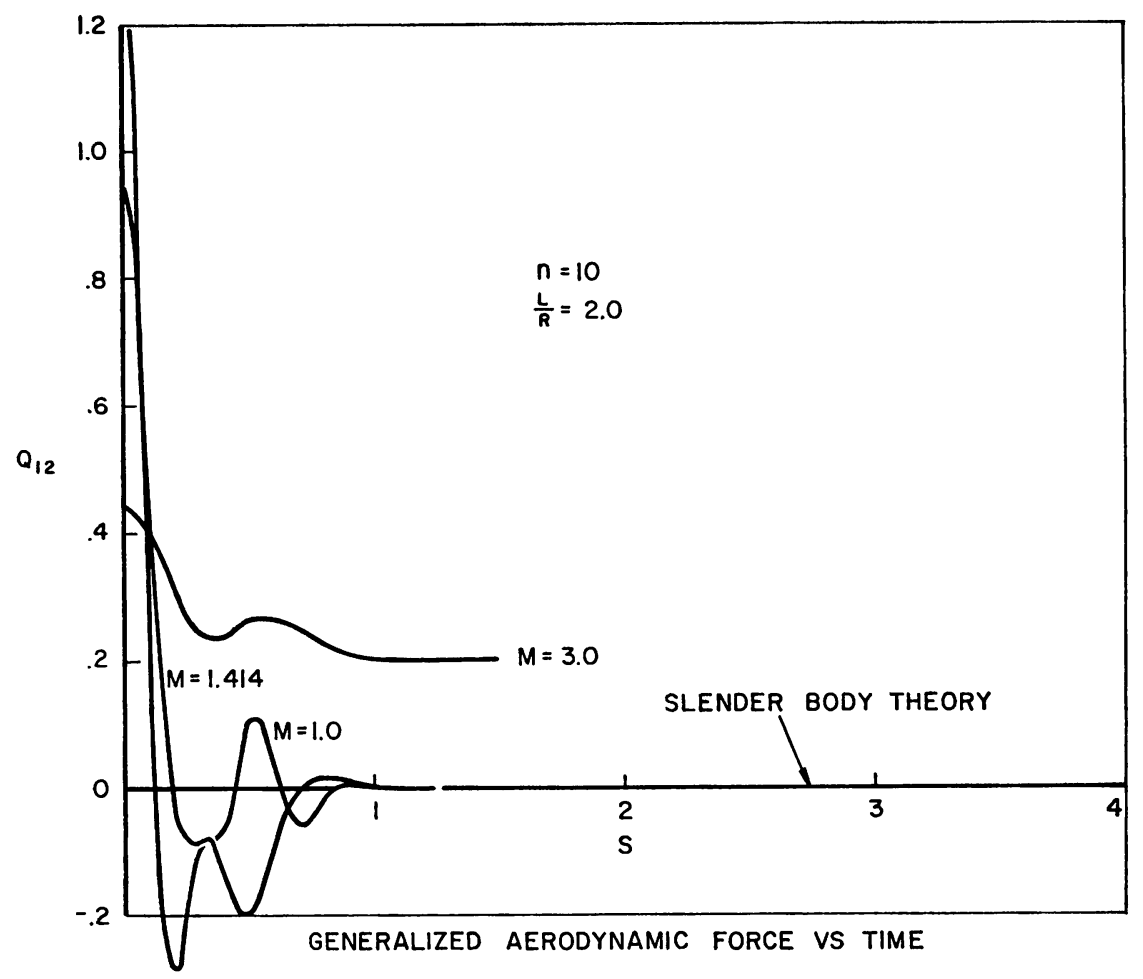

Finally, it should be emphasized that although the numerical results presented here are for the technologically important case of supersonic flow, the solution is equally valid and efficient for subsonic flow.

Acknowledgment. This work was supported by NASA Grant NGR31-001-059.

\section{REFERENCES}

[1] E. H. Dowell and S. E. Widnall, Generalized aerodynamic forces on an oscillating cylindrical shell, Quart. Appl. Math., 24, 1-17 (1966)

[2] E. H. Dowell and S. E. Widnall, Generalized aerodynamic forces on an oscillating cylindrical shell: Subsonic and supersonic flow, AIAA Journal (4) 4, 607-610 (April 1966)

[3] S. E. Widnall and E. H. Dowell, Aerodynamic forces on an oscillating cylindrical duct with an internal flow, J. Sound Vib., (1) 6, 113-127 (1967)

[4] D. G. Randall, Supersonic flow past quasi-cylindrical bodies of almost circular cross-section, ARC R. and M. No. 3067 (1958)

[5] R. Stearman, Research on panel flutler of cylindrical shells, Midwest Research Institute, AFOSR Report 64-0074 (1964)

[6] E. H. Dowell, Generalized aerodynamic forces on a flexible plate undergoing transient motion, Quart. Appl. Math. 24, 331-338 (1967)

[7] R. L. Bisplinghoff and H. Ashley, Principles of aeroelasticity, John Wiley and Sons, New York, 1962

[8] Bateman Manuscript Project, Tables of integral transforms, Vol. 1, McGraw-Hill, New York, 1954 